Ophthalmologe $2022 \cdot 119: 1280-1284$

https://doi.org/10.1007/s00347-022-01586-7

Eingegangen: 24. November 2021

Überarbeitet: 30. Dezember 2021

Angenommen: 24. Januar 2022

Online publiziert: 1. März 2022

(c) Der/die Autor(en) 2022

\section{Aderhautischämie als einzige ophthalmologische Manifestation einer Riesenzellarteriitis}

\author{
Martin Dominik Leclaire (D) · Justus Obergassel · Julia Biermann \\ Klinik für Augenheilkunde, Universitätsklinikum Münster, Münster, Deutschland
}

\section{Anamnese und klinischer Befund}

Eine 76-jährige Patientin stellte sich im Notdienst aufgrund einer deutlichen, 14 Tage zuvor akut eingetretenen Sehverschlechterung vor. Bei einer selbst durchgeführten Überprüfung sei ihr nach Zuhalten des linken Auges ein deutlich schlechteres Sehen rechts aufgefallen. Auf Nachfrage berichtete die Patientin von bitemporalen Kopfschmerzen mit Kämmschmerz und linksseitigen Kauschmerzen seit etwa 3 Wochen. An bekannten Allgemeinerkrankungen lagen eine arterielle Hypertonie und eine Hypothyreose vor. Gewichtsverlust, Muskel- oder Bewegungsschmerzen verneinte die Patientin. Auf Einweisung des Hausarztes hin befand sich die Patientin aufgrund der Kopfschmerzen und des Visusverlusts bei initialem Verdacht auf Schlaganfall bereits 3 Tage in stationärer, neurologischer Abklärung inklusive kranieller MRT und Dopplersonographie der extra- und intrakraniellen Gefäße. Die Abklärung hatte keinen Anhalt für einen zerebralen Infarkt ergeben, eine ebenfalls durchgeführte Blutdruckmessung über $24 \mathrm{~h}$ hatte hypertone Werte in den Tagstunden (im Durchschnitt 160/75 mm Hg [mm Quecksilbersäule]) erbracht.

Bei der notfallmäßigen Vorstellung betrug der unkorrigierte Visus rechts Fingerzählen und links 1,0, der intraokulare Druck betrug applanatorisch rechts 10 und links $12 \mathrm{~mm} \mathrm{Hg}$. Spaltlampenbiomikroskopisch präsentierte sich beidseitig eine reizfreie Pseudophakie bei deutlichem relativem afferentem Pupillendefekt am rechten Auge. Fundoskopisch zeigte sich rechts eine randscharfe Papille mit einer Exkavation von 0,3 mit partieller, temporal betonter Abblassung ohne Schwellung oder Blutungen, die Netzhaut zeigte sich bis auf eine diskrete Abschattung mit Farbveränderung nach temporal unauffällig. Am linken Auge wies die Papille keine Auffälligkeiten auf, die Netzhaut zeigte bis auf einen Aderhautnävus superior keine Auffälligkeiten. Die initiale Blutuntersuchung ergab ein erhöhtes CRP von $3,2 \mathrm{mg} / \mathrm{dl}$ (Normwert <0,5 mg/dl) sowie eine leichte Thrombozytose von $494 \mathrm{tsd} / \mu \mathrm{l}$ (Normwert 173-390tsd/ $\mu \mathrm{l}$ ). Die BSG lag bei $20 \mathrm{~mm}$ nach $1 \mathrm{~h}$ und bei $34 \mathrm{~mm}$ nach $2 \mathrm{~h}$. Die A. temporalis superficialis war im Seitenvergleich rechtsseitig nur schwach pulsierend tastbar. Es lag aber keine Verhärtung oder Prominenz der Arterie vor. Es erfolgte eine stationäre Aufnahme bei Verdacht auf Riesenzellarteriitis, und eine intravenöse Steroidtherapie mit Prednisolon $250 \mathrm{mg} / \mathrm{Tag}$ wurde initiiert. Am Folgetag erfolgte eine weitergehende Abklärung: In der multimodalen Bildgebung ergaben sich in der optischen Kohärenztomographie eine Aderhautverdickung sowie Alterationen der äußeren Netzhautbanden (-Abb. 1), in der optischen Kohärenztomographie (OCT) der Papille eine grenzwertige Nervenfaserschichtdicke inferior (- Abb. 2). Die Fluoreszein- und die Indocyaningrünangiographie zeigten eine großflächige Aderhautischämie (- Abb. 3). Die $30^{\circ}$-Schwellenperimetrie zeigte einen nasal betonten großflächigen Gesichtsfelddefekt (Mean Deviation -23,54 Dezibel; • Abb. 4). 


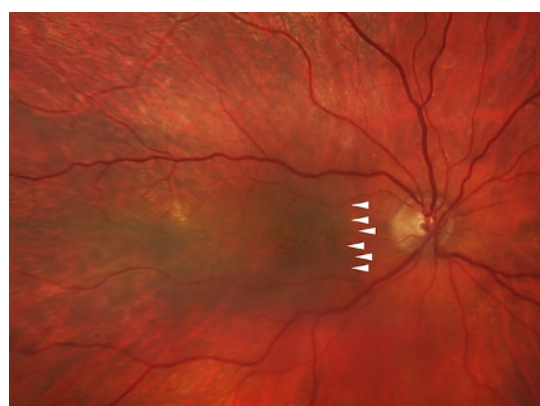

Abb. $1 \Delta$ Fundusbefund des rechten Auges am Tag nach der notfallmäßigen stationären Aufnahme. Es zeigte sich eine fundoskopisch sehr diskret imponierende Pigmentverdichtung zentral, die zur Papille hin recht scharf abgegrenzt war (Pfeilspitzen). Die Verfärbung korrespondiert in ihrer Ausdehnung nach nasal mit der in der Fluoreszenzangiographie sichtbaren Aderhautischämie (vgl. - Abb. 3)

\section{Diagnose}

Wir stellten die Diagnose einer Aderhautischämie bei Verdacht auf Riesenzellarteriitis.

\section{Therapie und Verlauf}

Am 2. stationären Tag erfolgte eine Biopsie der A.temporalis superficialis rechts, da der bildmorphologische Nachweis eindeutiger Vaskulitiszeichen nicht gelang. Histopathologisch zeigte sich mikroskopisch eine ausgeprägte Intimafibrose mit einem nahezu transmuralen überwiegend lymphoplasmazellulären Entzündungszellinfiltrat mit Nachweis von zahlreichen mehrkernigen Riesenzellen im Sinne einer typischen riesenzellhaltigen Arteriitis passend zu einer Arteriitis temporalis.

Die intravenöse Steroidtherapie wurde am Folgetag nach der stationären Aufnahme mit einer gesteigerten Dosis von $500 \mathrm{mg}$ Prednisolon pro Tag fortgeführt. Hierunter normalisierte sich das CRP am fünften stationären Tag, sodass die Entlassung erfolgen konnte. Der Visus und die $30^{\circ}$-Schwellenperimetrie waren bei Entlassung beidseitig stabil (Funktion unkorrigiert rechtes Auge Fingerzählen, linkes Auge 1,0), Sehbeschwerden am linken Auge wurden zu keinem Zeitpunkt geäußert.

Die Steroide wurden oralisiert (initial $60 \mathrm{mg}$ pro Tag, begleitende Magenulkus-, Infektions- und Osteoporoseprophylaxe mit Pantoprazol $40 \mathrm{mg} / \mathrm{Tag}$, Vitamin D $1000 \mathrm{IE} / \mathrm{Tag}$, Cotrimoxazol $480 \mathrm{mg} 3$-mal

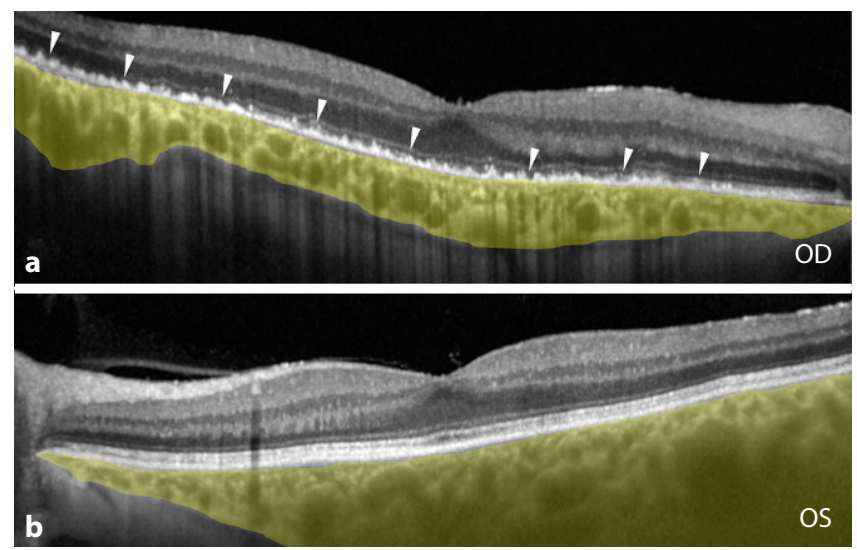

Abb. 2 \ Spectral domain-optische Kohärenztomographie des rechten Auges (OD, a) und des linken Auges $(\mathrm{OS}, \mathbf{b})$. Am rechten Auge zeigten sich eine insbesondere im Seitenvergleich imponierende Verdünnung der Aderhaut (gelb markierte Flächen) sowie Alterationen im Bereich des retinalen Pigmentepithels und der Photorezeptorbande (Pfeilspitzen). Die inneren Netzhautschichten zeigten sich hingegen regelrecht organisiert

wöchentlich und Amphotericin B $100 \mathrm{mg}$ 4-mal täglich) und das Reduktionsschema einer Steroidmonotherapie nach EULARAlgorithmus 2018 verordnet [6]. Die Steroidtherapie wurde unter der oben genannten Supportivtherapie problemlos vertragen. Auf eine sofortige zusätzliche steroidsparende Therapie mit Tocilizumab wurde bei gutem Ansprechen auf Steroide entsprechend den aktuellen Empfehlungen verzichtet. In einer Verlaufskontrolle nach 7 Wochen zeigte sich (unter einer täglichen Steroiddosis von $25 \mathrm{mg}$ Prednisolon/Tag) die Aderhautischämie fluoreszenzangiographisch stabil zum Vorbefund, hausärztliche CRP-Kontrollen waren zwischenzeitlich weiter unauffällig, Visus und Fundusbefund blieben ohne Veränderung, weiterhin bestanden keine Beschwerden am Partnerauge.

In einer weiteren Verlaufskontrolle 6 Monate nach dem stationären Aufenthalt imponierten fundoskopisch Pigmentverklumpungen im Bereich der Makula, das OCT zeigte zunehmende Alterationen des Pigmentepithels und der äußeren Netzhautbanden (• Abb. 5). Der Visus besserte sich auf bestkorrigiert 0,02 am rechten Auge.

\section{Diskussion}

Die Riesenzellarteriitis (RZA) ist die häufigste Vaskulitis bei Patienten über 50 Jahren und hat eine steigende Inzidenz mit steigendem Lebensalter [12]. Sie ist häufig mit einer Polymyalgia rheumatica assozi- iert [4]. Es handelt sich um eine granulomatöse Vaskulitis [1], betroffen sind hauptsächlich die kraniellen Abgänge der Aorta und hier insbesondere die A. temporalis, die A. ophthalmica, die Aa. ciliares posteriores sowie die A. vertebralis [3]. Da darüber hinaus auch weitere Gefäße betroffen sein können und auch andere Vaskulitiden die A. temporalis befallen können, wurde die früher gebräuchliche und auch heute immer noch häufig synonym verwendete Bezeichnung Arteriitis temporalis inzwischen verlassen [5]. Ein erhöhtes CRP und eine erhöhte BSG weisen eine hohe Sensitivität $(>80 \%$ ) bezüglich des Vorliegens einer histologisch nachweisbaren RZA auf, allerdings gibt es auch Fälle von RZA, bei denen sowohl BSG als auch CRP normwertig sind. In einer retrospektiven Analyse traf dies auf $4 \%$ der RZA-Patienten zu [8]. In dem hier präsentierten Fall war lediglich das CRP erhöht bei normwertiger BSG. Dies zeigt, dass eine normwertige BSG allein eine RZA nicht ausschließt.

Die häufigsten okulären Manifestationen einer RZA sind in abnehmender Häufigkeit die arteriitische anteriore ischämische Optikusneuropathie (arteriitische AION), ein retinaler arterieller Gefäßverschluss und die arteriitische posteriore ischämische Optikusneuropathie (arteriitische PION) [7].

Die arterielle Versorgung der Papille erfolgt im Wesentlichen aus den Aa. ciliares posteriores breves und den kurzen Ästen der A. centralis retinae, die beide aus $\operatorname{der} A$. ophthalmica entspringen und 

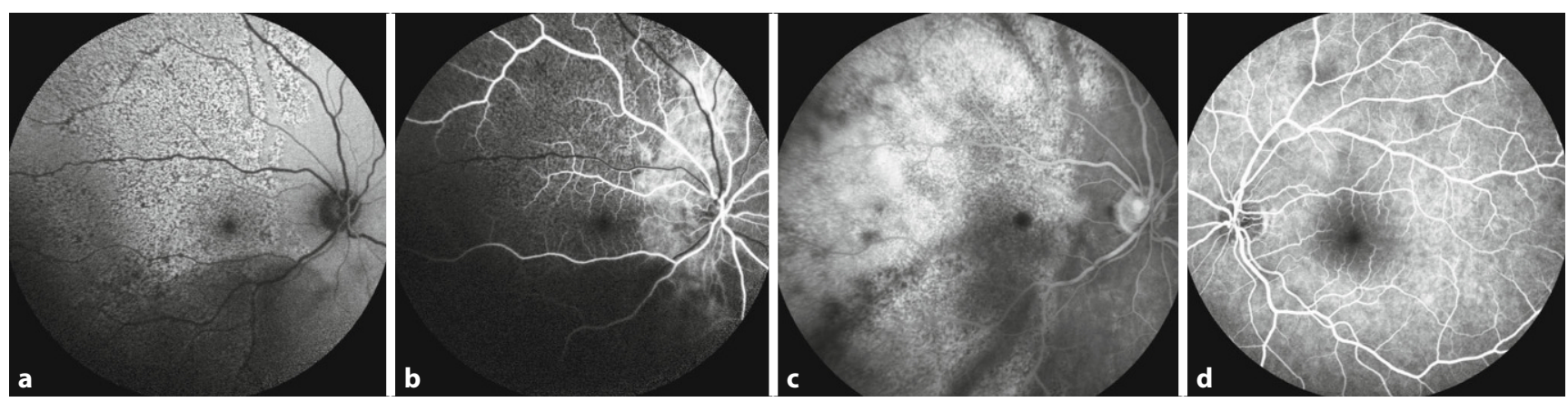

Abb. $3 \Delta$ a-c Fluoreszeinangiographie (FAG) des rechten Auges in der Frühphase (a), in der frühen arteriovenösen Phase (b) und in der Spätphase (c). Es imponiert eine großflächige Aderhautischämie. d Zum Vergleich die FAG des linken Auges in der arteriovenösen Phase

Schwellentest, zentral 30-2

Fixationskontr.: Blinder Fleck

Fixationsmarke: Zentral

Fixationsverluste: $10 / 13 x x$

Falsch POS-Fehler: $6 \%$

Falsch NEG-Fehler: $0 \%$

Testdauer: 07:04

Fovea: AUS

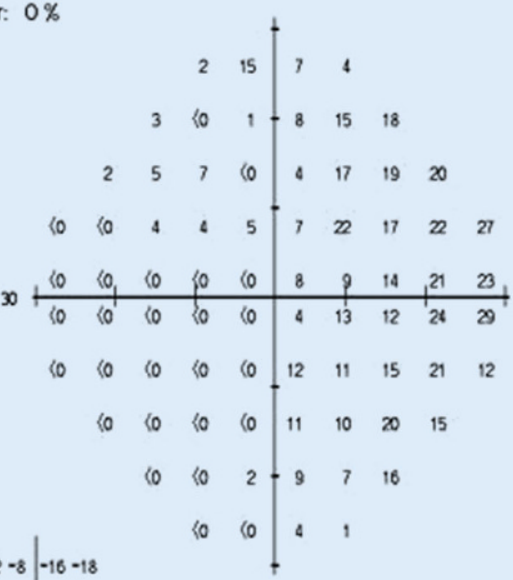

$-22-8 \mid-16-18$

$-23-28-25-18-10-7$

$-25-22-21-30-24-11-8-6$

$-27-30-28-27-25-23-7-11-60$

\begin{tabular}{llll}
$-28-31-32-33-33-23-22$ & -7 & -6 \\
\hline$-28-31-33-34-34$ & $-27-18$ & -5 & 0
\end{tabular}

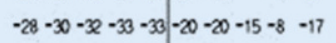

$-29-31-32-32-19-20-9-14$

$-29-30-28-20-20-13$

$-28-20 \mid-24-27$
Stimulus: III, WeiB

Hintergnund: 31.5 ASB

Strategie: SITA-Fast
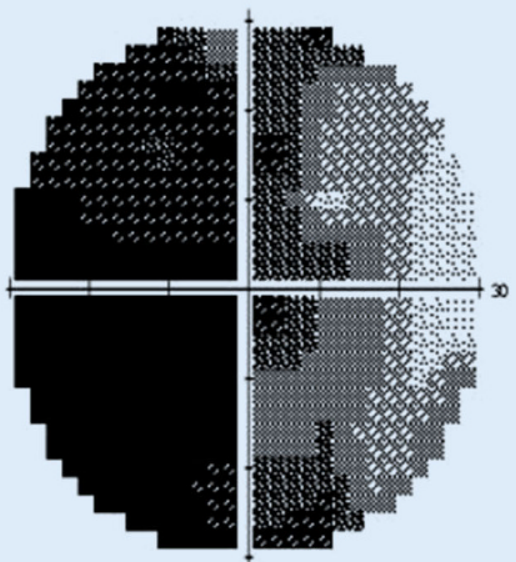

Korrigierte Abweichung

wird fur stark

abgeschwăchte Felder

nicht angezeigt. Siehe

"Gesamtabweichung" w Testzuverlassigkeit gering ***

GHT

AuBerhalb normaler Grenzen

VFI $20 \%$

MD $\quad-23.54 \mathrm{~dB} P<0.5 \%$

PSD $10.19 \mathrm{~dB} P<0.5 \%$
Abb. $4 \varangle$ Initiale $30^{\circ}$ Schwellenperimetrie am Tag nach der stationären Aufnahme mit großfächigem, nasal betontem Gesichtsfelddefekt im Bereich der Papille Anastomosen bilden (Zinn-Haller-Gefäßkranz). Die Perfusion der Choroidea entspringt ebenfalls aus den Aa. ciliares posteriores breves, weshalb eine verminderte choroidale Perfusion im Rahmen der RZA plausibel erscheint. Eine deutlich verringerte choroidale Füllung bei arteriitischer AION ist bereits in früheren Studien beschrieben worden, und flächige nicht perfundierte choroidale Areale wurden als ein Unterscheidungsmerkmal zwi- schen der arteriitischen und der nichtarteriitischen AION postuliert. Ferner konnten Pellegrini et al. in einer OCT-Studie einen verringerten "choroidal vascularity index" (entspricht dem Verhältnis der Fläche der choroidalen Gefäßlumina zur Gesamtfläche der Choroidea im makulären SD-OCT) bei Patienten mit arteriitischer AION im Vergleich zu gesunden Kontrollen und im Vergleich zu Patienten mit nichtarteriitischer AION zeigen ( $n=20$ in jeder Gruppe, $p=0,002$ bzw. $p<0,001$ ) [11]. Das Auftreten einer Aderhautischämie im Rahmen einer Riesenzellarteriitis wurde in mehreren Fallberichten beschrieben $[2,9,10]$, scheint jedoch deutlich seltener als die arteriitische AION oder der arterielle retinale Gefäßverschluss aufzutreten. Kopsachilis et al. berichten von einer zentralen Aderhautischämie bei einem 70-jährigen männlichen Patienten im Rahmen einer bioptisch gesicherten Riesenzellarteriitis [9]. 

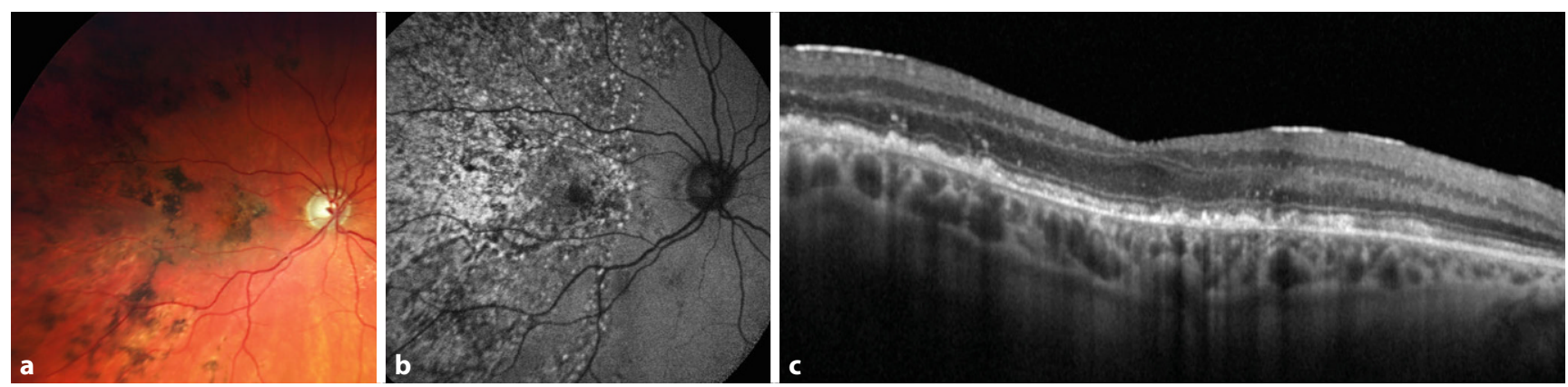

Abb. 5 ム Befund 6 Monate nach der initialen Vorstellung am rechten Auge. a Zentrale Fundusfotografie der Pigmentverklumpungen, $\mathbf{b}$ Fundusautofluoreszenz des gleichen Bereiches, c Spectral domain-optische Kohärenztomographie mit sich deutlich darstellenden Alterationen des Pigmentepithels und der äußeren Netzhautbanden

Ähnlich wie in dem von uns präsentierten Fall zeigten sich bei dem von Kopsachilis et al. vorgestellten Patienten im Verlauf von 6 Monaten zunehmende chorioretinale Narben bei leicht verbessertem Visus auf dem betroffenen Auge. Aufgrund der Zunahme der Alterationen des Pigmentepithels und der äußeren Netzhautbanden scheint es plausibler, diesen Visusanstieg einer exzentrischen Fixation anstatt einer chorioretinalen Funktionsverbesserung in dem betroffenen Areal zuzuschreiben. Eine weitere, ähnlich konfigurierte zentrale Aderhautischämie (65-jährige Patientin, weiblich) wird von Olali et al. referiert [10]. Im Gegensatz zu diesen Fällen präsentierte sich die Aderhautischämie bei unserer Patientin nicht lokalisiert im Bereich der Makula, sondern deutlich großflächiger (बAbb. 2). In einem weiteren Fallbericht wird von einer 71-jährigen Patientin berichtet, die im Rahmen einer RZA eine großflächige Aderhautischämie entwickelte, allerdings trat diese bilateral auf, und es zeigten sich beidseitig Cotton-Wool-Herde als Zeichen einer Mitbeteiligung der A. centralis retinae [2]. In keinem der 3 hier erwähnten Fälle wird eine gleichzeitig vorliegende AION oder ein retinaler Gefäßverschluss beschrieben.

Die Tatsache, dass in der Literatur zu dem Thema bislang nur Fallberichte und keine Studien bzw. Metaanalysen existieren, deutet auf die Seltenheit dieser Manifestation der RZA hin. Die Fallberichte zeigen eine Heterogenität des Ausmaßes der Ischämie von begrenzten makulären bis hin zu großflächigen beidseitigen Befunden. Aufgrund der fundoskopisch sehr diskreten Veränderungen ( $\bullet$ Abb. 1$)$ ist die Diagnosestellung schwieriger als bei der arteriitischen AION oder dem arteriellen Gefäßverschluss. Daher spielt die Anamnese eine wichtige Rolle und insbesondere bei älteren Patienten mit plötzlicher Visusverschlechterung sollte dezidiert nach einer typischen RZA-Klinik (Kopfschmerz, Kauschmerz, Kämmschmerz, B-Symptomatik) sowie nach Symptomen einer Polymyalgia rheumatica gefragt werden. Auch der Tastbefund der Temporalarterien kann weitere Hinweise auf das Vorliegen einer RZA liefern. Die Bestimmung von CRP und BSG sollte umgehend erfolgen. Eine Fluoreszeinangiographie ist für die Diagnosestellung erforderlich und sollte bei Sehstörungen im Rahmen einer vermuteten oder gesicherten RZA insbesondere dann durchgeführt werden, wenn fundoskopisch keine Zeichen für eine AION oder einen Gefäßverschluss vorliegen. Die umgehende Einleitung einer hoch dosierten Steroidtherapie ist zur Prophylaxe einer Manifestation am Partnerauge essenziell. Dennoch kommt es in 34-75\% der RZAFällezu einem Rezidiv unter ausschleichender Steroidtherapie, weshalb regelmäßige Verlaufskontrollen der Patienten essenziell sind und die Patienten aufgeklärt werden sollten, dass bei Auftreten subjektiver Beschwerden wie einer Sehverschlechterung eine umgehende ophthalmologische Kontrolle erfolgen sollte. Im Falle eines Rezidivs sollte eine ergänzende Therapie mit Tocilizumab oder alternativ Methotrexat erwogen werden [6].

\section{Zusammenfassung}

Eine Aderhautischämie ist eine mögliche, wenngleich seltene ophthalmologische Manifestation der RZA, die im Rahmen der Fundoskopie nicht in Erscheinung tritt und daher übersehen werden kann. Neben der häufigeren arteriitischen AION und dem arteriellen Gefäßverschluss sollte an diese mögliche Manifestation gedacht werden und eine arteriitische Genese insbesondere bei älteren Patienten mit akuter und subakuter Visusminderung auch bei zunächst weitgehend unauffälligem Papillen- und Netzhautbefund ausgeschlossen werden. Die Kenntnis und Bewertung der Aderhautischämie als Zeichen einer RZA ermöglichen die umgehende Behandlung mit Steroiden und beugen einer weiteren Verschlechterung oder Erblindung vor.

\section{Korrespondenzadresse}

\section{Dr. med. Martin Dominik Leclaire}

Klinik für Augenheilkunde, Universitätsklinikum Münster

Domagkstr. 15, 48149 Münster, Deutschland martindominik.leclaire@ukmuenster.de

Funding. Open Access funding enabled and organized by Projekt DEAL.

\section{Einhaltung ethischer Richtlinien}

Interessenkonflikt. M.D. Leclaire, J. Obergassel und J. Biermann geben an, dass kein Interessenkonflikt besteht.

Für diesen Beitrag wurden von den Autoren keine Studien an Menschen oder Tieren durchgeführt. Für die aufgeführten Studien gelten die jeweils dort angegebenen ethischen Richtlinien. Für Bildmaterial oder anderweitige Angaben innerhalb des Manuskripts, über die Patienten zu identifizieren sind, liegt von ihnen und/oder ihren gesetzlichen Vertretern eine schriftliche Einwilligung vor.

Open Access. Dieser Artikel wird unter der Creative Commons Namensnennung 4.0 International Lizenz veröffentlicht, welche die Nutzung, Vervielfältigung, 
Bearbeitung, Verbreitung und Wiedergabe in jeglichem Medium und Format erlaubt, sofern Sie den/die ursprünglichen Autor(en) und die Quelle ordnungsgemäß nennen, einen Link zur Creative Commons Lizenz beifügen und angeben, ob Änderungen vorgenommen wurden.

Die in diesem Artikel enthaltenen Bilder und sonstiges Drittmaterial unterliegen ebenfalls der genannten Creative Commons Lizenz, sofern sich aus der Abbildungslegende nichts anderes ergibt. Sofern das betreffende Material nicht unter der genannten Creative Commons Lizenz steht und die betreffende Handlung nicht nach gesetzlichen Vorschriften erlaubt ist, ist für die oben aufgeführten Weiterverwendungen des Materials die Einwilligung des jeweiligen Rechteinhabers einzuholen.

Weitere Details zur Lizenz entnehmen Sie bitte der Lizenzinformation auf http://creativecommons.org/ licenses/by/4.0/deed.de.

\section{Literatur}

1. Buttgereit F, Dejaco C, Matteson EL, Dasgupta B (2016) Polymyalgia rheumatica and giant cell Arteritis: a systematic review. JAMA 315:2442-2458. https://doi.org/10.1001/jama.2016.5444

2. Cohen S, Gardner F (2006) Bilateral choroidal ischemia in giant cell arteritis. Arch Ophthalmol 124:922. https://doi.org/10.1001/archopht.124.6. 922

3. Gajree S, Borooah S, Dhillon N et al (2017) Temporal artery biopsies in South-East Scotland: a fiveyear review. J R Coll Physicians Edinb 47:124-128. https://doi.org/10.4997/JRCPE.2017.203

4. Gonzalez-Gay MA (2004) Giant cell arteritis and polymyalgia rheumatica: two different but often overlapping conditions. Semin Arthritis Rheum 33:289-293. https://doi.org/10.1016/j.semarthrit. 2003.09.007

5. Hayreh SS, Podhajsky PA, Zimmerman B (1998) Ocular manifestations of giant cell arteritis. Am J Ophthalmol 125:509-520. https://doi.org/10. 1016/s0002-9394(99)80192-5

6. Hellmich B, Agueda A, Monti S et al (2020) 2018 Update of the EULAR recommendations for the management of large vessel vasculitis. Ann Rheum Dis 79:19-30. https://doi.org/10.1136/ annrheumdis-2019-215672

7. Jennette JC, Falk RJ, Andrassy K et al (1994) Nomenclature of systemic vasculitides. Proposal of an international consensus conference. Arthritis Rheum 37:187-192. https://doi.org/10.1002/art. 1780370206

8. Kermani TA, Schmidt J, Crowson CS et al (2012) Utility of erythrocyte sedimentation rate and C-reactive protein for the diagnosis of giant cell arteritis. Semin Arthritis Rheum 41:866-871. https://doi.org/10.1016/j.semarthrit.2011.10.005

9. Kopsachilis N, Pefkianaki M, Marinescu A, Sivaprasad $S$ (2013) Giant cell arteritis presenting as choroidal infarction. Case Rep Ophthalmol Med. https://doi.org/10.1155/2013/597398

10. Olali C, Aggarwal S, Ahmed S, Gupta M (2011) Giant cell arteritis presenting as macular choroidal ischaemia. Eye 25:121-123. https://doi.org/10. 1038/eye.2010.169

11. Pellegrini M, Giannaccare G, Bernabei Fet al (2019) Choroidal vascularity index in arteritic versus nonarteritic anterior Ischemic optic neuropathy. Invest Ophthalmol Vis Sci 60:2275-2275
12. Weyand CM, Goronzy JJ (2009) Medium- and large-vessel vasculitis. https://doi.org/10.1056/ NEJMra022694.Zugegriffen:28. Feb. 2022
DOG

Deutsche Ophthalmologische Gesellschaft

Gesellschaft

für Augenheilkunde

\section{Neu gegründete Sektion \\ DOG-Ophthalmologische \\ Onkologie sucht Mitglieder}

Die Deutsche Ophthalmologische Gesellschaft (DOG) initiiert die Gründung einer Sektion für Augentumoren. Die „Sektion DOG-Ophthalmologische Onkologie" soll künftig alle Aktivitäten und Expertisen auf diesem Spezialgebiet der Augenheilkunde bündeln, Fortschritte in Wissenschaft und Therapie fördern und sich für die Bedürfnisse der Patientinnen und Patienten einsetzen.

Ziel der neuen Sektion soll es sein, alle Aktivitäten auf dem Gebiet der intraund periokulären sowie orbitalen gutund bösartigen Tumorerkrankungen zu koordinieren. Das umfasst die Fort- und Weiterbildung durch Symposien und Kurse sowie die Erarbeitung von Forschungsschwerpunkten, von Behandlungsempfehlungen und Leitlinien. Dazu gehört ferner der onkologisch-interdisziplinäre Erfahrungsaustausch sowie die Erarbeitung aktueller Behandlungsstrategien in Zusammenarbeit mit nicht-ophthalmologischen onkologischen Fachgesellschaften. Weitere Betätigungsfelder sind der Austausch mit überregionalen Fachorganisationen wie der International Society of Ocular Oncology (ISOO) oder der Ophthalmic Oncology Group (OOG), die Mitarbeit in Bündnissen wie der Nationalen Dekade gegen Krebs oder dem UV-Schutzbündnis sowie die Förderung von Netzwerken für klinische Strukturmaßnahmen, Studien und Grundlagenforschung.

Gesucht werden nun DOG-Mitglieder mit einem fachlichen Bezug zu den Tumoren am und im Auge, die sich in der neuen Sektion engagieren möchten. Augenärztinnen und Augenärzte, die beitreten möchten, können sich über den OnlineMitgliedsbereich der DOG für die Sektion anmelden: https://www.dog.org/diedog/sektion-dog-ophthalmologischeonkologie/ 